\title{
Formulación y evaluación de proyectos. Enfoque para emprendedores
}

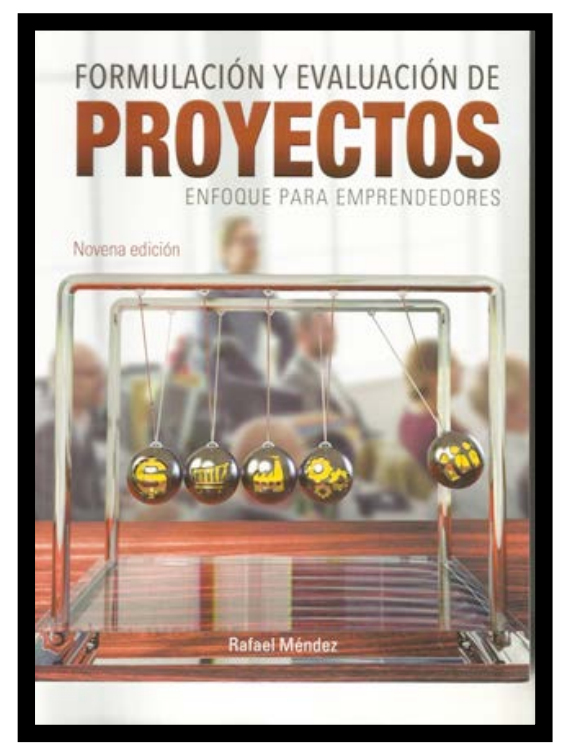

Méndez, Rafael. (2016). Formulación y evaluación de proyectos. Enfoque para emprendedores. Bogotá.

Toda persona está en capacidad de crear proyectos productivos, pero en general se carece de una metodología que le permita examinar las variables pertinentes para definir la conveniencia o no de participar en un proyecto de inversión, o en un proyecto de desarrollo en el campo social, cultural, deportivo o ambiental.

En las instituciones y empresas es evidente la necesidad de promover y consolidar una cultura de los proyectos para alcanzar mejores niveles de competitividad.

El reacomodamientos geopolítico mundial, los procesos de globalización de la economía, la conformación de grandes bloques de mercados, las firmas de tlc, los avances tecnológicos, así como la importancia del conocimiento y la información como armas estratégicas del desarrollo, constituyen escenarios atractivos para la identificación de nuevos proyectos de desarrollo.
De otra parte el reciclamiento de las funciones del estado, la descentralización de los entes territoriales y la participación de la sociedad civil, implican la definición de áreas estratégicas para mejorar la calidad de vida de la comunidad; y la forma de materializar esas estrategias es mediante los planes, programas y proyectos de desarrollo.

Esta obra ofrece un marco de referencia sistemático para identificar, formular y evaluar un proyecto de inversión en cualquier sector de la economía sea público, privado o de carácter misto, independientemente de su tamaño.

\section{¿Por qué se hizo el libro?}

El libro "formulación y evaluación de proyectos. Enfoque para emprendedores", surgió ante la necesidad de estudiantes de pregrado y posgrados en diferentes áreas del conocimiento de tener una guía metodológica que les permitiera preparar, formular y evaluar con un enfoque sistemático un proyecto en el nivel de prefactibilidad o de factibilidad, estimulando a la vez creatividad y la mentalidad empresarial, 
como requisito para la gestión de proyectos exitosos en cualquier sector de la economía. de igual manera, es una respuesta para los empresarios que con frecuencia tienen que sustentar sus proyectos antes agentes externos para acceder a fuentes de financiación y apoyo.

La gran aceptación de las ocho ediciones anteriores en instituciones de educación superior y la consulta permanente de empresarios y directivos de instituciones públicas y privadas, motivaron la publicación de esta nueva edición revisada, corregida, actualizada y ampliada, teniendo en consideración las dificultades de quienes incursionan en el proceso de ampliación, modernización, reestructuración de una unidad productiva existente o en la creación de una nueva organización productiva de viene y/o servicios.

\section{¿Qué lo diferencia de otros libros?}

Existen varios factores diferenciadores de este libro, con respecto a otros textos disponibles en el mercado. Algunos de ellos son:

- Permite de una manera sencilla, entender y aplicar los conceptos básicos en el proceso de preparación, formulación y evaluación de un proyecto en el nivel de prefactibilidad o de factibilidad en cualquier sector de la economía, sin sacrificar el nivel de profundidad requerido.

- Motiva a un pensamiento reflexivo, creativo e innovador, aprovechando la experiencia de quien o quienes inician el proceso de elaboración de un estudio de prefactibilidad o de factibilidad.

- La forma en que se presenta la secuencia e interacción entre los diferentes componentes de un proyecto, le permite al lector entender la importancia de trabajar con un enfoque sistemático y comprender la relación simbólica y de mutua dependencia entre los diversos componentes del proyecto.

- Elsitio web que complementa al librocontiene ejemplos de tablas-guías que ilustran sobre los contenidos para diferentes tipos de proyectos; ejemplos de instrumentos de encuestas para captar información de campo. además, contiene casos de iniciativas emprendedoras exitosas, un ejemplo de cooperación internacional, un aplicativo financiero y estadístico, así como otras herramientas de gran utilidad para quienes elaboran un proyecto.

- La sección "gerencia su proyecto" contiene recomendaciones prácticas con base en la experiencia del autor e incluye un instrumento de evaluación del proceso del preparación y formulación de un proyecto de prefactibilidad o de factibilidad, que permite al responsable del proyecto autoevaluar el cumplimiento de los requisitos básicos cuando se elabora cada componente del proyecto y retroalimentar si es necesario este proceso también es de especial utilidad para las entidades que cumplen funciones de conceptos sobre estudios presentados.

- Es el único libro en el área de proyectos que incorpora dos aplicaciones informáticas. la primera llamada evaproyect, que permite al lector elaborar los estados financieros del proyecto hasta obtener los indicadores integrales de rentabilidad: tasa interna de rendimiento (tir); valor presente neto (vpn) y relación beneficio costo $(b / c)$, cesamiento estadístico (spe), facilita procesar encuestas y elaborar simultáneamente proyectos de investigación.

- Incorpora temas de especial interés hoy en día para los proyectos, tales como: normas ISO 9000 y entidades sin ánimo de lucro y estructuración e proyectos con base en las etapas del ciclo de proyectos desde la perspectiva de la metodología de marco lógico $(\mathrm{mml})$, ampliamente utilizada para la presentación de proyectos de cooperación internacional.

- Desarrolla el concepto del rol de la gerencia en el ciclo del proyecto de naturaleza pública o privada e identifica desarrollos tecnológicos para gerencia de proyectos.

- Las figuras que acompañan el libro y la cuidadosa interrelación de los componentes estudiados en cada capítulo, estimulan en el lector la creatividad para el diseño de los instrumentos requeridos y orientar con éxito la formulación de un proyecto.

- En los capítulos 3 a 9 se incluyen casos de análisis que corresponden a entornos de la realidad latinoamericana.

- Comprender la relación entre un estudio de factibilidad y un plan de empresa es fundamental para participar con éxito en los concursos 
regionales, nacionales e internacionales relacionados con la creatividad, la innovación y el emprendimiento. por ellos, se incluye esta temática en el capítulo 11.

- La creación de nuevas unidades productivas basada en la aplicación de los conceptos presentados en el libro tanto por estudiantes de pregrado como de posgrado, así como la gestión de nuevos recursos por parte de los empresarios, es la mayor evidencia de la funcionalidad y campo de aplicación del libro.

- Entender la relación entre la investigación, la innovadora y el emprendimiento de alto impacto, es un elemento diferenciado con respecto a otras obras disponibles en el mercado.

\section{¿Cuáles son sus contenidos?}

El libro está conformado por 13 capítulos que le permiten al lector comprender los conceptos básicos que se deben tener en cuenta, antes de iniciar el proceso de preparación, formulación y evaluación de un proyecto. gradualmente aborda el desarrollo cada uno de los componentes del proyecto, siempre teniendo en cuenta que la visión, creatividad, experiencia y rigurosidad en la aplicación de los conceptos estudiados por parte de quien elabora el estudio, es lo que permite generar la diferenciación cuando se concibe la creación de un proyecto productivo de bienes y/o servicios en cualquier sector de la economía, independientemente que la fuentes de recursos sea el sector público, privado o una combinación de los dos.

El capítulo 1 presenta algunas de las condiciones que son críticas para contextualizar un proyecto, establece la relación entre el plan, el programa y el proyecto y orienta sobra la importancia del espíritu emprendedor como condición para construir una sociedad sana, capaz, de generar crecimiento económico, social y una paz duradera.

De igual manera, se ilustra sobre las fuentes de identificación de proyectos y sobre cómo seleccionar la mejor alternativa para desarrollar un proyecto, los actores que participan en las diferentes etapas del proyecto, el concepto de horizonte del proyecto y recomendaciones de carácter práctico cuando se quiere "vender" la idea de un proyecto.

Aclara la importancia de los semilleros de investigación, los grupos de investigación y la generación de ideas que puedan conducir a proyectos de emprendimientos asociados a la innovación y el desarrollo tecnológico.

El capítulo 2 ofrece al lector una visión de conjunto de los elementos e interrelaciones de cada uno de los componentes del proyecto. Antes de iniciar la elaboración del mismo conviene leer con atención este capítulo, ya que suministra al responsable del proyecto elementos de juicio para planificar la ejecución de las actividades asociadas al proyecto y mantenerse ubicado cuando realice cada componente. También incluye un diagrama integral de la interrelación de los diferentes componentes del proyecto.

Los capítulos 3, 4, 5, 6, 7, 8, 9 y 10 abordan de manera sistemática casa componente del proyecto, desde el estudio de mercado y comercialización o análisis de oferta demanda hasta la evaluación financiera y deimpacto social, pasando por los aspectos técnicos (localización, tamaño e ingeniería de proyecto), los aspectos organizativos y las inversiones y presupuestos de ingresos, costos y gastos relacionados con la fase operativa del proyecto.

El capítulo 1 trata sobre los estudios de pre-inversión y las características del plan de empresa. Este documento es importante, pues las entidades que financian a los emprendedores lo exigen. Además, se brindan todas las orientaciones para sustentarlo exitosamente.

El 12 aborda la temática acerca de proyectos de cooperación internacional con la metodología de marco lógico $(\mathrm{mml})$, que exigen los organismos bilaterales y multilaterales de cooperación, las ONG y el sector público para asignar recursos del estado.

El capítulo 13 "introducción a la generación de proyectos" se concibo para comprender y dimensionar el verdadero rol del gerente de proyectos, en los sectores público y privado. 


\section{¿A quiénes va dirigido?}

Este libro se destina a quienes consideran que el desarrollo no se logra con grandes saltos sino que el resultado del conocimiento de la propia realidad, de la selección de áreas estratégicas para transformar esa realidad y de la canalización del conocimiento, el talento y la energía humana para mejorar sus condiciones de vida.

\section{Investigadores y educadores}

El libro constituye un marco de referencia sistemático del proceso de formulación $\mathrm{y}$ evaluación de un proyecto, para quienes tiene la convicción de la necesidad de reciclar los procesos educativos y de orientar la investigación hacia la solución de problemas concretos de la sociedad. En tal sentido, se puede utilizar como texto primario en combinación con libros especializados en cada uno de los temas tratados.

\section{Directivos}

La calidad de las decisiones en entidades públicas y privadas depende de la capacidad de identificar las variables clave que determinan la oportunidad y el ciclo de la vida de un proyecto, así como del conocimiento y experiencia en la aplicación de las técnicas de evaluación de esas variables.

El libro es de especial utilidad para los directivos, cuando se ven enfrentados a tomar decisiones sobre la conveniencia que su organización participe en el desarrollo de un proyecto.

\section{Gerentes de proyectos}

El ejecutivo en la dirección de proyectos encontrará en este libro un instrumento útil para diseñar propuestas técnicas y para programar las actividades relacionadas con los equipos interdisciplinarios que participan en la formulación y evaluación de un proyecto. Aunque cada proyecto tiene su propia especificidad, los componentes y la metodología de trabajo pueden ser examinados en detalle siguiendo paso a paso cada capítulo.

\section{Líderes empresariales y comunitarios}

Eléxito de un proyecto depende del compromiso efectivo de cada uno de los actores interesados en su desarrollo y de la calidad del estudio en su conjunto. Hoy existen múltiples fuentes de financiación de proyectos tales como los fondos de cofinanciación, el fondo financiero de proyecto de desarrollo (fonade), e incluso organismos de cooperación internacional entre otras.

El acceso a esos recursos está estrechamente ligado con la presentación de proyectos debidamente sustentados. El libro en su primera parte permite a los líderes empresariales y comunitarios, comprender las claves para presentar proyectos exitosos; posteriormente, desarrolla con mayor nivel de profundidad cada componente del proyecto en su lenguaje comprensible.

\section{Estudiantes}

Uno de los mayores retos de las entidades educativos es formar generaciones con la mentalidad empresarial y con capacidad para generar calor agregado a la sociedad. El libro orienta al estudiante de pregrado y posgrado, sobre cómo integrar los conocimientos en torno a una idea con potencial desarrollo empresarial, pues está comprobado que cualquier disciplina del conocimiento pueden surgir iniciativas empresariales $\mathrm{y}$, este libro constituye una herramienta útil para acompañar la consolidación de un espíritu emprendedor.

\section{Evaluadores de proyectos}

Una de las dificultades más frecuentes para los evaluadores de un proyecto s la falta de un instrumento ágil, sistemático y coherente para abordar la evaluación de cada una de las áreas que conforman un proyecto en el nivel de prefactibilidad o de factibilidad. Por esta razón, en cada capítulo se incluye la sección "gerencie su proyecto", que permite autoevaluar el proceso de formulación de un proyecto y aportar elementos de evaluación que pueden ser adaptados de manera creativa a las necesidades específicas de las entidades evaluadas para elaborar sus instrumentos de análisis de proyectos. 